\title{
Interview with Mike Kosterlitz
}

\author{
Patrícia F.N. Faísca1 and Rui D.M. Travasso ${ }^{2}$ - Dol: https://doi.org/10.1051/epn/201931 \\ $\square{ }^{1}$ Departament of Physics and BiolSI - Biosystems and Integrative Sciences Institute, Faculty of Sciences, University of Lisboa, Campo \\ Grande, Ed. C8, 1749-016 Lisboa, Portugal \\ - ${ }^{2}$ Department of Physics and CFisUC - Centro de Física da Universidade de Coimbra, Faculty of Sciences and Technology, University of \\ Coimbra, R. Larga, 3004-526 Coimbra, Portugal
}

\section{In the summer of 2018, Professor Michael Kosterlitz visited Portugal as a plenary speaker of the FíSICA2018 conference organised by the Portuguese Physical Society (SPF) and by the University of Beira Interior. FÍSICA 2018 comprised two meetings: the $28^{\text {th }}$ lberian Meeting for Physics Teaching, and the $21^{\text {st }}$ National Conference of Physics, a biannual event that brings together researchers from all areas of Physics working in Portugal.}

ÍSICA 2018 was a fruitful encounter between researchers, high-school teachers and students interested in sharing experiences and in discussing the state-of-the-art of research in Physics. Professor Kosterlitz's talk entitled “Topological defects and phase transitions - Vortices and dislocations (A random walk through physics to a Nobel Prize)" was integrated in the $21^{\text {st }} \mathrm{Na}$ tional Conference of Physics.

During his visit, Professor Kosterlitz was interviewed by Patrícia Faísca, from the Department of Physics and BioISI (University of Lisboa), and by Rui Travasso from the Department of Physics and CFisUC (University of Coimbra).

\section{Vita}

Professor Kosterlitz is currently the Harrison E. Farnsworth Professor of Physics at Brown University, where he has been teaching and doing research since 1982. He was born in Aberdeen (Scotland, UK) in 1943. He studied at the University of Cambridge (1961-1966), and did a DPhil in high-energy physics at the University of Oxford (1969). He then went to the University of Birmingham, and decided to move into the field of condensed matter physics, working as a post-doc with David Thouless (1970-1973). Together they published a paper [1] on phase transitions in two-dimensional systems (in the XY-model), where they introduced a novel type of long-range order based on the overall properties of the system (instead of the twopoint correlation function), and to which they referred as topological order. The latter gives rise to an unusual phase transition, known as the Kosterlitz-Thouless (KT) transition, which underlies the superfluid transition in ${ }^{4} \mathrm{He}$ films. The importance of this work was recognized with the Nobel Prize in Physics in 2016, which Professor Kosterlitz (prize share: 1/4) and Duncan Haldane (prize share: 1/4) shared with David Thouless (prize share: $1 / 2$ ). Prior to receiving the Nobel Prize his contributions to Physics were recognized with the Lars Onsager Prize (2000), and with the Maxwell Medal (1981). Professor Kosterlitz is a passionate mountaineer and in his youth he was considered one of the best climbers in Britain. In 2017 he was awarded the prestigious Climbing Ambassador prize at the Arco Rock Legends Awards. This prize recognizes "those who, through their passion, energy and vision have guided and influenced the development of this sport".
1. How did the environment in your household while you were a kid drive your curiosity towards science?

I guess you could say my parents didn't try to influence what I studied at all. I gravitated naturally towards maths and science because I have a lousy memory, and all the humanity subjects required too much memory. So I didn't handle them very well. But I found maths and science didn't require too much memory. And I could handle them with logic and deduction, which suited my way of functioning.

\section{In line with what you have just said, in your Nobel biography [2] you mention that you could do best in Physics and Mathe- matics than in Chemistry because your "ability to make logical deductions compensated for your unreliable memory". Was this the main reason why you decided to become a physicist?}

I think so. One of the other reasons was that although I really like Chemistry I decided it was too dangerous. One time I was just holding a test tube of a colourless liquid, wondering what to do with it... Then I noticed it was changing colour for no reason at all, so held it out away and then boom! It exploded. So I decided, Chemistry is not for me, it's too dangerous. Chemistry was fun because I could mostly control when something exploded, but then I realized there were some things I couldn't control. So I decided, no, this is too dangerous. Theoretical Physics is much safer.

\section{What, in your opinion, are the main advantages of taking a degree in Physics? Do you take the view that a background in Physics is the best to develop problem-solving abilities?}

Yes, I suppose that Physics is about the only subject in which you learn how to solve problems. In Mathematics the things you learn are about problems that are already solved, whereas in Physics any problem is given as a set of words disguising a problem, and one learns how to understand the problem and to solve it. So I think Physics is a good subject to learn for this reason.

4. In 2017, i.e., one year after being awarded the Nobel Prize in Physics, you received the Climbing Ambassador at the Arco Rock Legends Awards. How do you rate the importance of this prize? To me it is as important as the Nobel Prize. 
5. We understand that it is not straightforward to explain the KT phase transition to the general public since it requires a substantial amount of Physics background. Do you teach it to your students? How would you summarise it?

I don't teach it to undergraduates, because I think it's probably too sophisticated for the undergraduate level. But I do teach it as an advanced graduate course. If you happen to be interested in phase transitions, and you want to understand why a thin helium film goes from a super fluid to a normal fluid, then I would say that the only way to understand how a super flow can dissipate is through the creation of vortices and so, the KT theory is all about the properties of a collection of vortices in two dimensions. The good thing about two dimensions is that vortices are points, which is why Thouless and I studied this problem in two dimensions. David said vortices are important and complicated in three-dimensions and so, look at the problem in two-dimensions.

\section{Regarding the presence of KT transitions in} many different 2D systems (from XY-models to superfluids, from Bose-Einstein condensates to melting) which recent systems that have KT transitions would you highlight?

I think one of the most interesting applications is recent stuff on cold gases: cold atoms, clouds of cold atoms. If you make a two-dimensional system of this it is possible to have a KT transition in this system too, and surprisingly, the theory fits fairly well with the experimental data, although not as well as it does in superfluid or in two-dimensional melting. These transitions are associated with exactly the same Physics as superfluid helium, but the cloud is too small to really get good data from it. a very clear explanation, and from that point on, we went on very well together, and the rest is history. I was quite surprised because I was very nervous about going to Thouless's office because he had a reputation for, how should I say... not suffering fools gladly. And by his standards almost everybody else was a fool. So I knew that when I went up to talk to him I was leaving myself open to appearing like a complete idiot. But fortunately he made this mistake, and I decided in the future that every time I didn't understand him, I would assume he had done the same thing. And this worked. So we actually got on very well together.

\section{Since you did not come from condensed matter Physics, you didn't realise at that time, or, at least, did not realise properly, the extent of the contribution that you were making, right?}

No, no idea. To Thouless and I it was just an interesting theoretical problem that needed a solution, and that was the only reason we did it. A fascinating theoretical problem.

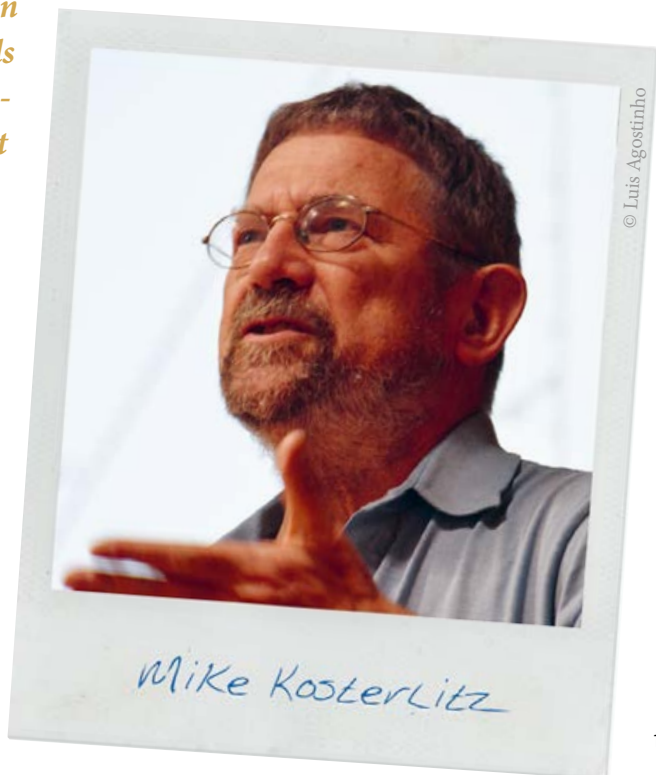

9. At what point did the impact hit you? As I said, it was my first foray into condensed matter Physics, and I thought this is what Physics should be. Something new, some new ideas, and the possibility to put everything together and come up with a result. Thouless said that what we had done was very good and should be important but that was all we thought about it. We just knew we'd done something good. We wrote it up, had some trouble getting it published, because it was just too new. Eventually, as I discovered later, one referee just said 'I don't understand this stuff' but he let it through and it was published. It was not cited for the first 5-6 years - not even a single citation - and then

7. At the time, during your work in the KT transition, did you have the feeling of discovery? Was it obvious to you that the new type of transition would have a large impact in the community? You have to remember that this was the first problem in condensed matter that I was involved in. I was a high-energy physicist before that, and I was getting very, very fed up with it because I was not getting anywhere. I was doing long, elaborate calculations basically for nothing. I got really tired of it when I was in Birmingham and I decided that I had to find another problem. So I was walking around the department asking everybody 'Do you have a problem that I may look at? Because I need something new'. And the answer in general was 'no' until I got to David Thouless's office. I asked him the question, and then I spent the next couple of hours in his office listening to him talking about various phenomena, staring at the board, writing equations and things. I was understanding very little of it. Eventually I got to admit I was not understanding a word of what he was talking about, and I thought I was looking like a complete idiot. So, I said, 'David, I'm sorry I have to stop you there. Could you please explain where the first equation that you wrote down came from?' He turned and said 'Didn't I tell you that?' And I could honestly say 'No, you didn't'. And he said 'Oh!' and then he proceeded to give me suddenly, the citations started to come. Then Halperin and Nelson basically re-did the theory in a much simpler way, and basically came up with the same results, and then it took off.

10. In a recent review on $K T$ Physics [3] you say that at the time you moved from high energy Physics to condensed matter Physics you were most of all searching for an interesting (but tractable) problem. Nowadays, in an era where fundable research should have a clear societal impact, working on an interesting problem is not necessarily the route for a successful career in science. Do you think that the current funding policies have a negative impact on the development of science and scientific progress?

Yes and no. Scientific progress needs money so that people can actually do the research. Therefore, the funding agencies are actually important for providing support for scientists. However, the funding agencies are normally interested - as you said - in more directed research rather than letting the researchers do what they want to do. And directed research is unlikely to produce a major breakthrough. The major breakthroughs are going to come from curiosity driven research. But the chances of a major breakthrough 
coming from a piece of research are actually very small, and it is impossible to say, ok, let's fund this person because he or she is going to produce a breakthrough. It's more like playing the roulette, spinning the wheel, and saying ok, we'll bet several thousand dollars that this piece of research is going to be important. And the chances are that you're going to lose, but occasionally you'll be hitting the jackpot. But not very often. So, I don't know what should be done, because you have to pay a scientist to live, to be able to work and to produce original research, but just because you pay money doesn't mean that you are going to have that piece of original research coming out. So, I don't know how you can do it.

\section{In your Nobel biography [2] you state that during your stay in Cornell with Wilson and Fisher you came to learn "the im- portance of testing one's theory against the ultimate authority in Physics: experiment". As you know, several theoretical physicists (especially string theorists, cosmologists, but also some high ener- gy physicists) are strong advocates of the principle that "elegance will suffice" (i.e. if a theory is sufficiently elegant and explanatory, it need not be tested experimentally). Do you consider (e.g. as George Ellis and Joe Silk [4]) that the integrity of Physics is at risk and should be defended?}

A theory like string theory has produced essentially no new Physics. It has produced some important advances in pure Mathematics, and so on. So it has not been a waste of time. But I wouldn't agree with the statement that a theory does not have to have experimental proof because a theory without an experimental verification is exactly what? It's cold. It's a theory. It's nothing more than that. The way I look at Physics is: we live in a Universe that does its own thing. It does not care about your Mathematics; it does not care about my Mathematics. It does what it does. And our job is to try to explain, or to understand why the Universe is doing what it does. The best tool we have is Mathematics. A piece of Mathematics is a self-contained consistent scheme. And, in principle, within that scheme all the answers are contained in this piece of self-consistent Mathematics. But this whole mathematical structure has to be compared to the real world. And maybe it will work. Maybe it will agree with the real world. But more likely it won't. And I think that this is the relation between Mathematics and Physics, and the real world. And maybe it's the right structure to describe the real world, or maybe it is.

12. Can you tell us a little bit about your current research interests? The problem that I've been trying to understand is a problem in non-equilibrium Physics. Many driven out-of-equilibrium systems do seem to come to some sort of stationary state, a time-independent state. I'm looking at a system that does have a deterministic part, and does have a set of stationary states. Then you put some noise onto it. Just simple additive noise. And I'm asking the question: Does a unique stationary state get picked out on average? It's exactly what happens when a system evolves to equilibrium. We do know that this is true, that it does come to some equilibrium, some unique stationary distribution. And this unique stationary distribution is picked out by the thermal fluctuations or stochastic noise. And I was thinking, maybe the same thing happens for a driven out-of-equilibrium system, that some stochastic noise is essential to pick out a unique stationary state. Or at least, some narrow band of stationary states. Numerically this seems to be what happens, at least in some simple systems. But when you try to go through the Mathematics of this... For a system approaching equilibrium you write down a Langevin equation and asymptotically it comes to the Boltzmann distribution, and you can go through the Mathematics and demonstrate this - not rigorously - but demonstrate this. For the out-of-equilibrium system, which looks very similar, you can go through the same piece of Mathematics, but suddenly you discover that everything falls apart. And that's the problem I'm trying to understand.

13. Many Physics graduates will end up working as part of interdisciplinary teams researching topics that may be outside the traditional realms of Physics. How do you think we can best prepare our students for this new reality?

Well, I don't know how one can't teach such things, but I always thought that the connections between Physics and other fields are obvious, and shouldn't require much teaching. Basically, I knew this almost as a child - it's almost part of me that I know that Physics is connected with all sorts of other subjects, and has something to offer other fields. Some other fields, not all. And vice-versa. Maybe it has something to do with the way it was taught at school, when we were taught science. Sometimes it was Physics, sometimes Chemistry, or Biochemistry, and all these subjects were not really separated-out. They were all science. I still feel that way, that there shouldn't be any distinction between Physics, Chemistry and so on and so forth. They are all the same thing.

\section{Let us imagine that you were about to enter the University right now. Would you still choose to study Physics?}

Probably. Or may be not. I might choose to study something to do with computers, or a bit more mathematical. In fact at one point of my education, at Cambridge, I actually explored the possibility of dropping Physics and study Mathematics. But I was told, no, that is not a good idea, it is very difficult to change and, quite right because I probably would have managed Mathematics, but I think I would have got very irritated with that. As far as Physics is concerned, there are two ways of doing it. One is to have a very broad curriculum and learn essentially nothing about anything. The other way is to be more specialized and go more deeply into it. But that means you learn something about a very small subset of Physics and nothing about anything else. It is a choice that has to be made, and whichever choice you make, I don't think matters very much. Someone who is motivated enough will learn things not formally taught. And they are probably more successful people.

\section{References}

[1] J.M. Kosterlitz and D.J. Thouless, J Phys C: Solid State Phys 6, 1181-203 (1973)

[2] J. Michael Kosterlitz - Biographical. NobelPrize.org. Nobel Media AB 2018 (www.nobelprize.org/prizes/physics/2016/kosterlitz/auto-biography)

[3] J.M. Kosterlitz, Rep. Prog. Phys. 79, 026001 (2016)

[4] G. Ellis and J. Silk, Nature 516, 321 (2014) 